\title{
Dayside Auroral Particle Acceleration Mechanisms Derived from Dynamics Explorer Data
}

\author{
J. L. Burch ${ }^{1}$, J. D. Menietti ${ }^{1}$, and J. A. Slavin ${ }^{2}$ \\ ${ }^{1}$ Southwest Research Institute, San Antonio, TX 78228-0510, U.S.A. \\ ${ }^{2}$ NASA, Goddard Space Flight Center, Greenbelt, MD 2077I, U.S.A.
}

(Received November 17, 1989)

\begin{abstract}
Data from Dynamics Explorer hot-plasma and magnetic-field instruments have been used to investigate the characteristics of dayside electron acceleration regions, or inverted$V$ 's, and the nature of the field-aligned currents that flow in their vicinity. The data set examined in this study was obtained in the pre-noon sector, where the region-1 fieldaligned currents are typically flowing downward. Although not permanent features of the dayside auroral region, inverted- $V$ events are commonly observed to occur just equatorward of the cusp or cleft in a region which contains trapped electrons and which is believed to map to the boundary layer. The downward region-1 currents are observed either just poleward of the trapping boundary in the equatorward part of the cleft or, at times, within the same flux tubes as the inverted- $V$ events. In either location, the region- 1 currents are carried by accelerated ionospheric electrons flowing out of the ionosphere. Therefore, when the region- 1 currents flow through the inverted- $V$ events, both upward electron beams and upward ion beams are observed together. Although the inferred potential drops associated with the dayside inverted- $V$ events are about a factor of ten smaller than those observed on the night side, a downward field-aligned current is not consistent with common assumptions regarding the generation of auroral acceleration regions. Moreover, the existence of upward-accelerated ionospheric electrons within the inverted- $V$ events is inconsistent with their acceleration by parallel potential drops.
\end{abstract}

\section{Introduction}

The relationship between field-aligned currents and auroral particle acceleration has been the subject of numerous experimental and theoretical investigations over the past decade or so. A rather consistent picture has emerged in which field-aligned currents flow outward from the ionosphere within the acceleration regions while downward return currents flow along field lines in the adjacent regions. The dominant charge carriers of the field-aligned currents are the highly mobile electrons (MARSHALL et al., 1988), with accelerated auroral primary electrons providing the upward current and accelerated ionospheric electrons carrying the downward current. The expectation that intense auroral primary electron beams will dominate the direction of field-aligned current flow in any particular situation has led to the assumption that auroral arcs or inverted- $V$ 's occur only in association with upward currents, and several models have been based on this assumption (LYONS, 1980; CHIU et al., 1981).

In the study of MARSHALL et al. (1988), which considered nightside auroral oval data only, several patterns of field-aligned currents were observed around acceleration regions. The downward currents were, as expected, confined largely to the regions 
adjacent to inverted- $V$ 's, while upward currents were generally observed within inverted$V$ 's but were often more intense near the edges of the events and in some cases were extremely weak. In these cases of very weak field-aligned currents DE-1 was located above almost the entire region of acceleration, leading MARSHALL et al. (1988) to suggest a mid-altitude closure of currents under certain conditions.

In the study reported in this paper, the relationship between inverted- $V$ events and field-aligned currents was examined in the pre-noon sector of the polar magnetosphere. Analysis of a large number of polar passes of both DE-1 and DE-2 showed that by far the most common location of inverted- $V$ events, and indeed the only place where large-scale events were observed, is just equatorward of the polar cusp or cleft. This region is of particular interest in the pre-noon sector because there it typically contains downward region- 1 currents. Because of the continuity of the instantaneous auroral oval, it might be expected that inverted- $V$ events can occur in regions of large-scale downward fieldaligned currents. The alternatives in the morning sector would be for the inverted- $V$ 's to occur only at lower latitudes, in the dayside extension of the plasma sheet where the region-2 currents flow, or within the cusp region. Previous studies (e.g., SMIDDY et al., 1980) have demonstrated that the dayside region-1 currents close predominantly through the boundary layer. This observation, coupled with the fact that nightside inverted- $V$ events are associated with the plasma-sheet boundary layer, leads to the expectation that morning-sector inverted- $V$ 's might well contain downward field-aligned currents.

In this paper we consider several examples of morning-sector passes of the DE-1 spacecraft through the mid-altitude ( $\sim 2-3$ earth radii) cusp or cleft. We will show that dayside inverted- $V$ events are common features of the mid-altitude extension of the boundary layer, which lies just equatorward of the cusp. The downward region-1 fieldaligned currents flow through this same general region and often overlap the dayside inverted- $V$ events, although they often lie poleward of the inverted- $V$ 's in the equatorward reaches of the cusp itself. We find that upward-accelerated ionospheric electron beams are the carriers of downward region-1 currents even in regions of downward electron acceleration. Thus, quasi-static parallel potential drops are probably not responsible for acceleration of the ionospheric electrons. Some type of resonant wave acceleration is more likely.

\section{Observational Results}

Data from day 81270 (September 27, 1981). We begin by presenting data from a cusp pass that contained no discernible inverted- $V$ activity in order to illustrate the nature of the ion and electron distributions associated with the downward region-1 currents. A number of other examples of cusp region data from the DE-1 High Altitude Plasma Instrument (HAPI) have been published previously (e.g., BURCH et al., 1982, 1983; BURCH, 1988) so we will not repeat all of the features of the data presentation here. Instead we concentrate on the region of downward field-aligned current beginning at about 13:47 UT in Fig. 1. The energy-time spectrograms in Fig. 1 are plotted in a single-pitch-angle format in which only those data for pitch angles less than 10 degrees are plotted in the upper (electron) panel, while only data taken at particle pitch angles greater than 170 degrees are plotted in the middle (ion) panel. Thus, we present spectrograms for downward-moving electrons and upward-moving ions. The lower panel contains field-aligned current densities (positive upward) derived from the complete 
HAPI electron distribution functions. The ion currents are in all cases less than 1 per cent of the electron currents for this pass. Currents derived from the magnetometer data are also available and will be shown in some of the subsequent data presentations. As demonstrated by BURCH et al. (1983) and MARSHALl et al. (1988), at altitudes above about one earth radius there is consistently good agreement between the currents derived from the HAPI electron data and those derived from the magnetometer data from DE-1.

In Fig. 1 the downward region-1 currents are located just poleward of the apparent trapping boundary, which was crossed at approximately 13:47 UT, in a region of intense downward electron fluxes at energies near $100 \mathrm{eV}$ and upward ion fluxes at energies of several hundred $\mathrm{eV}$. After a brief gap in the strong fluxes, the main cusp plasma injection begins between 13:49 and 13:50 UT with the onset of strong upward cusp-region fieldaligned currents. The upward ion fluxes in the main injection region are composed of reflected cusp ions along with some ion conics (see, e.g., BURCH et al., 1982). However, the upward ion fluxes shown in Fig. 1 between 13:47 and 13:49 UT contain only very weak fluxes of cusp ions, if any. Also, even though there are significant downward electron fluxes in this time period, the electron distributions are dominated by upwardaccelerated ionospheric electron beams, which are responsible for the downward region-1 currents (BURCH et al., 1983). To illustrate the types of electron and ion distributions that are typically observed in the downward current regions in the absence of inverted- $V$ events we show an electron velocity-space contour plot in Fig. 2 and a similar plot for ions in Fig. 3. The upward electron beam that is responsible for the downward current is clearly discernible in Fig. 2, while a distinctive ion conic appears in the contour plot of Fig. 3. This coexistence of upward electron beams and ion conics has been reported before by KLUMPAR and HeikKILA (1982), HultQvist (1988), and HultQvist et al. (1988) and is consistent with models in which waves near the ion gyrofrequency can resonate with the gyro motion of ions and the parallel motion of electrons (see, e.g., the review by BURCH, 1990). Notable in Fig. 1, in addition to the absence of any significant inverted- $V$ events, is the fact that the region- 1 current system lies wholly within a dense low-energy plasma population that might be characterized as the cusp boundary layer (LiN et al., 1984) or as the outer part of the boundary layer on open field lines. Such a dense low-energy plasma population can easily supply large field-aligned current densities without the need for significant acceleration (KNIGHT, 1973).

Data from day 81278 (October 5, 1981). DE-1 data on day 81278 show a morphology characteristic of times when large-scale inverted- $V$ events occur in the late morning sector. Here the acceleration regions are located adjacent to and equatorward of the apparent keV-electron trapping boundary, which was crossed at approximately 13:51 UT. In Fig. 4, the energy fluxes of upward-moving electrons (top panel) and ions (middle panel) reveal the intense upward electron beams that carry the downward region-1 currents. The field-aligned current densities derived from the DE-1 magnetometer (MAGA) data are plotted in the bottom panel. There are at least two inverted- $V$ events in Fig. 4, one observed between about 13:44 and 13:46 UT and the other between 13:47 and 13:51 UT. Although the downward electron data are not shown, the acceleration events are quite evident in the ion data.

Figures 5 and 6 show electron and ion distribution-function, contour plots for a region within the first inverted- $V$. The electron distribution shows the same characteristics that are observed in nightside inverted- $V$ 's: the existence of a widened and deepened loss cone along the $-v_{\|}$axis, a hole distribution along the $+v_{\|}$axis at about $4500 \mathrm{~km} / \mathrm{s}$, a 


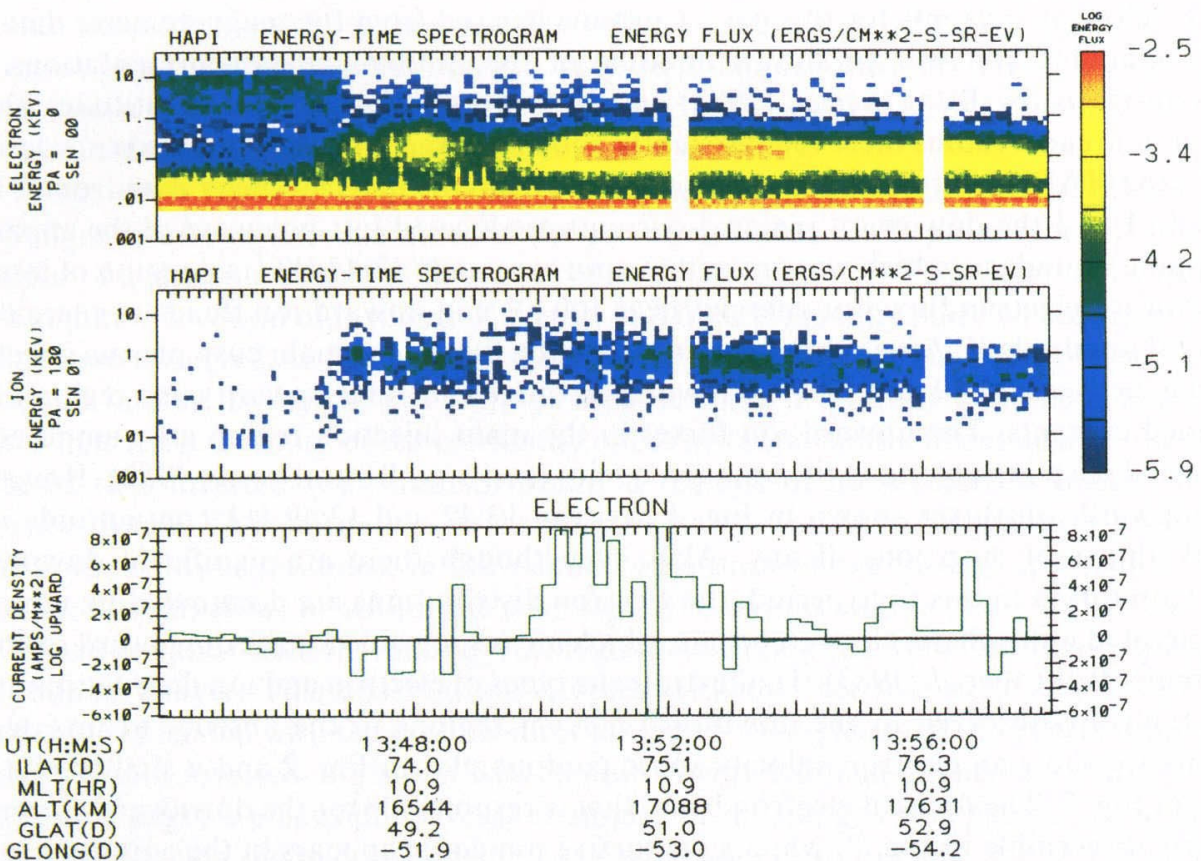

Fig. I. A three-panel plot of the DE-1 data for day 270 of 1981. Top: Energy-versus-time spectrogram with electron energy flux for precipitating electrons ( pitch angle $=0^{\circ}$ ) color-coded according to the color bar at the right: Middle: Same as top but for ions (pitch angle $=180^{\circ}$ ); Bottom: Current density (as determined from the HAPI particle data) versus time.

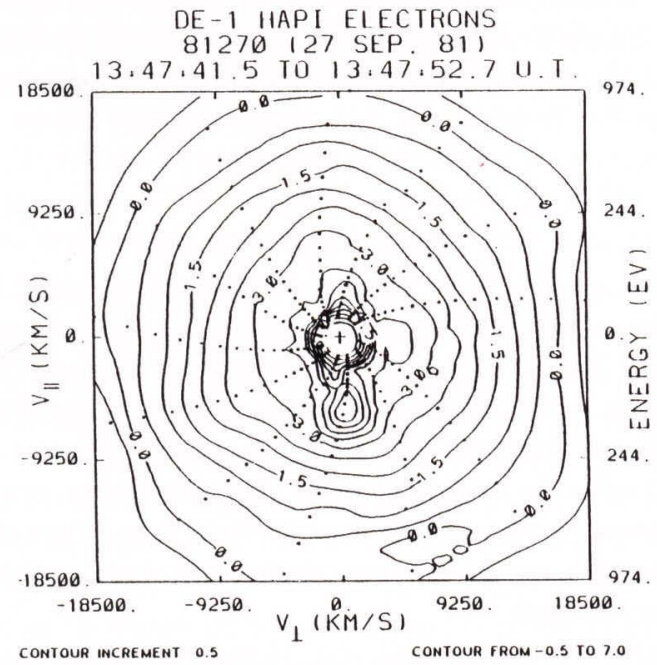

Fig. 2. Velocity space contours of the electron distribution. The data were obtained during 2 spins of the DE-1 satellite. Positive velocity is along the magnetic field (toward the Earth). The energy scale is highly non-linear in this and all the succeeding contour plots. 


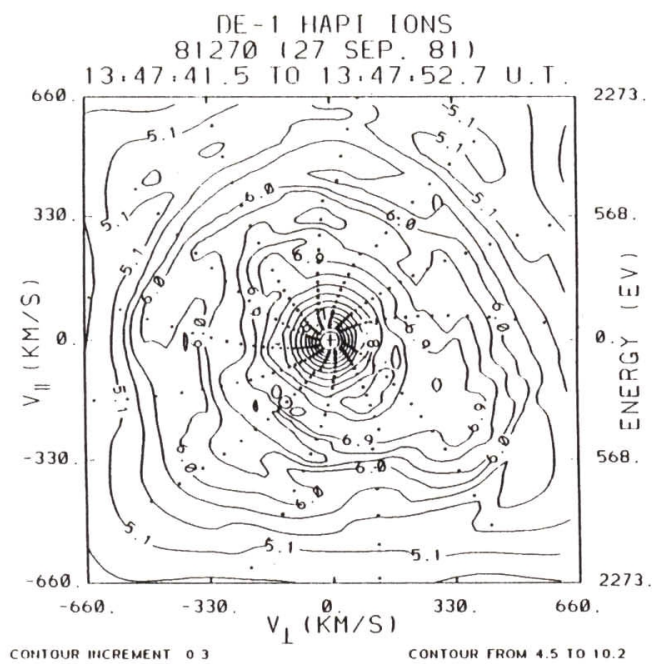

Fig. 3. Same as Fig. 2 except for positive ions.

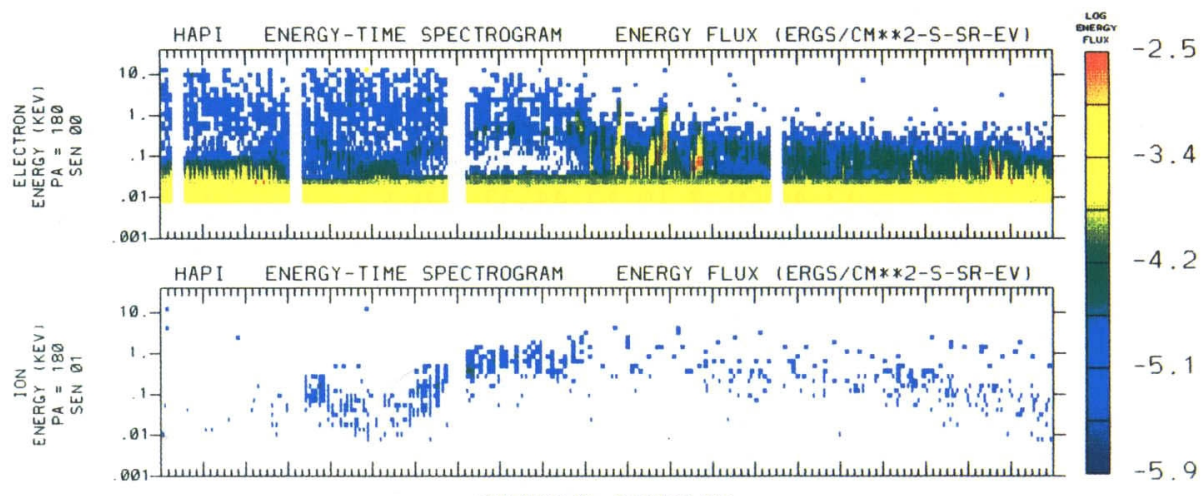
CURRENT DENSITY

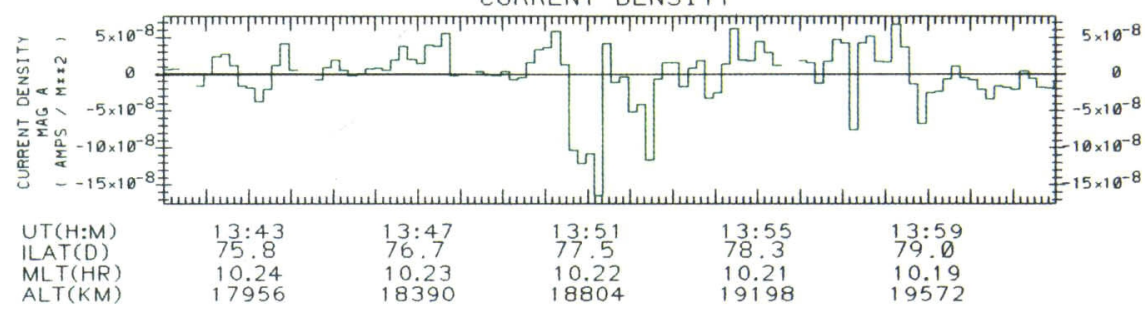

Fig. 4. A three-panel plot for day 278 of 1981 in the same format as Fig. 1 except that the current density in the bottom panel was calculated from the magnetometer data obtained from the MAGA instrument on board DE-I, and the spectrogram displays upward electron and upward ion energy fluxes. 


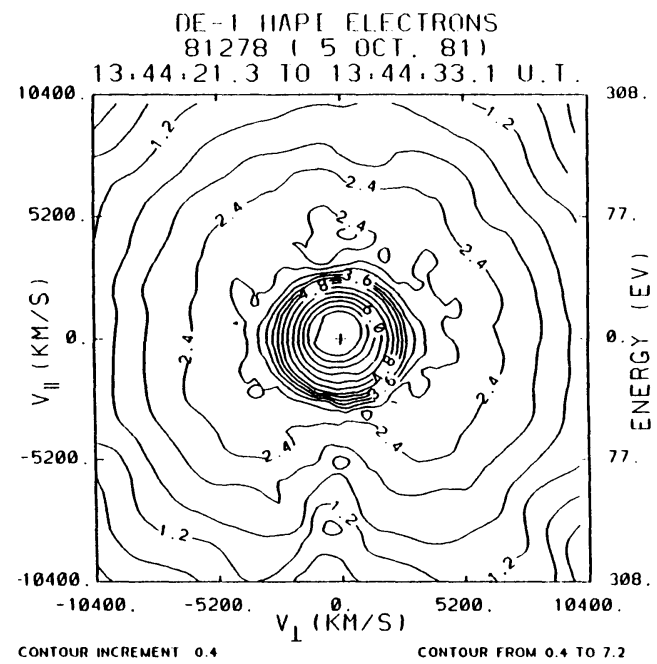

Fig. 5. Contours of the velocity-space distribution function for the electrons. This plot and that of Fig. 6 were obtained from data taken during the same two-spin period of the satellite. The electron distribution shows a hole feature at energies near $70 \mathrm{eV}$. There is also a well-developed loss cone feature.

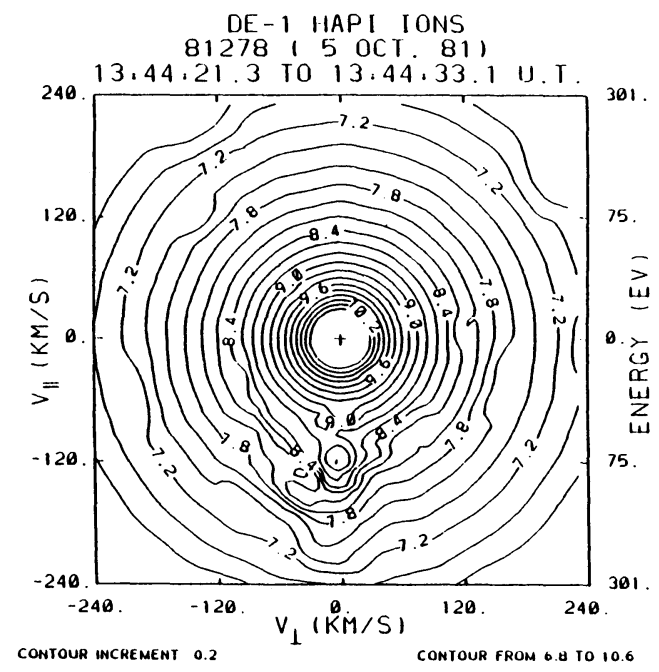

Fig. 6. Velocity space contours of the distribution function for ions. The main feature of the ions is a field-aligned beam (pitch angle $=180^{\circ}$ ) extending from less than $75 \mathrm{eV}$ to about $300 \mathrm{eV}$. 
nearly circular forbidden-region boundary defined approximately by the $\log _{10} f\left(\mathrm{~s}^{3} / \mathrm{km}^{6}\right)$ $=2.4$ contour, and a bump distribution centered along the $\pm v_{\perp}$ axis and partially filling the forbidden region. These features have been described by CHIU et al. (1983), OMIDI et al. (1984), and GURGIOLO and BURCH (1988) for distributions obtained at mid-altitudes over the nightside auroral oval. The ion distribution in Fig. 6 shows a well-defined upward-moving beam. Note, however, that the potential drops inferred for dayside inverted- $V$ 's (several hundred $\mathrm{eV}$ ) are generally quite small compared to those on the night side.

Although the field-aligned currents are rather weak in the region of the two inverted$V$ events in Fig. 4, they follow one of the characteristic patterns identified by MARSHALL et al. (1988): upward currents are observed near the edges of each inverted- $V$ with very weak currents in their interiors. However, there is an overlap of the more intense downward region-1 currents with the highest-latitude portion of the larger inverted- $V$ event extending from about 13:50:40 to 13:51:05 UT. This region, in fact, contains the most intense part of the region- 1 currents, which extend well poleward of the inverted- $V$ and occupy a region similar to that shown in Fig. 1. In Fig. 4, however, the beam-like nature of the electron distributions responsible for the downward currents is apparent because only the upward-moving electron fluxes are plotted. Figure 7 contains an electron distribution-function contour plot for the region of overlap between the region-1 currents and the higher-latitude inverted- $V$ event in Fig. 4 . As can be seen in Fig. 7, upward-accelerated beams of cold electrons are responsible for the downward currents in agreement with the previous results of BURCH et al. (1983), MENIETTI and BURCH (1987), and MARSHALL et al. (1988).

Data from day 81279 (October 6, 1981). Data from two successive orbits of DE-1 on October 6, 1981 are presented next as cases in which the downward region-1 currents occupy nearly the entire spatial region of inverted- $V$ events that are located just equatorward of the electron trapping boundary. An energy-time spectrogram in the usual

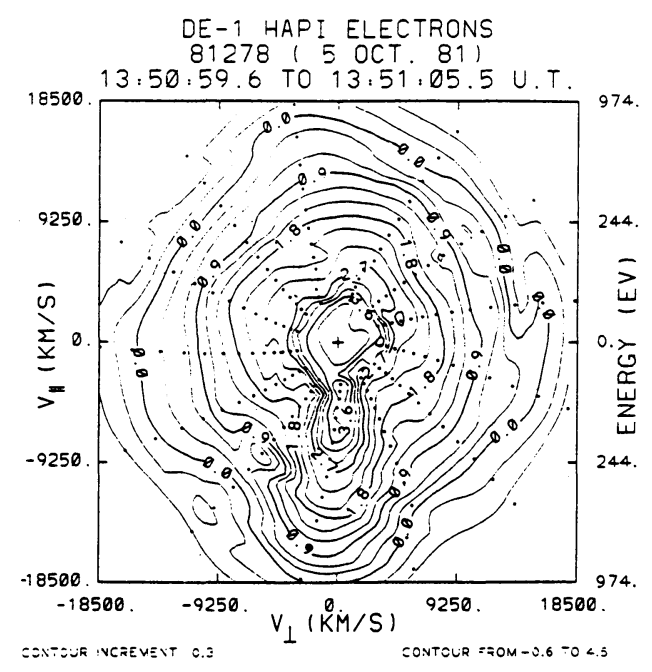

Fig. 7. A velocity-space contour plot of an electron distribution measured within the higher-latitude inverted$V$ event shown in Fig. 4. 


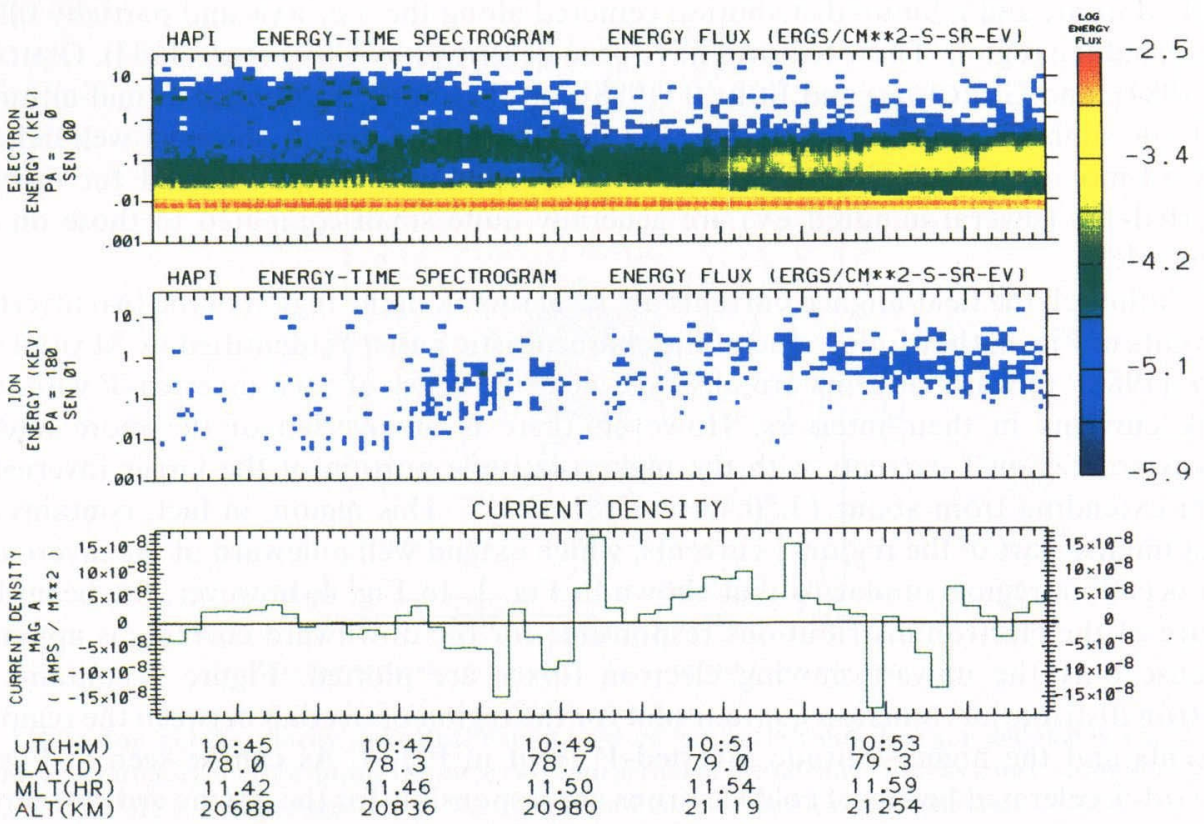

Fig. 8. A three-panel plot for day 279 of 1981 in the same format as Fig. 1 except that the field-aligned current densities are derived from the MAGA data.

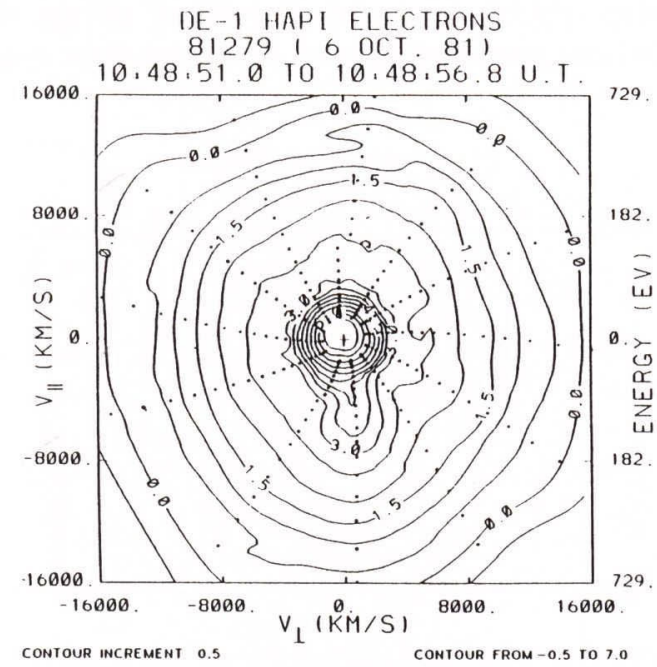

Fig. 9. Contours of the electron distribution function obtained from data taken during one spin of the satellite on day 279 of 1981 . There is a distinct field-aligned (pitch angle centered around $180^{\circ}$ ) electron beam that extends to about $180 \mathrm{eV}$. 


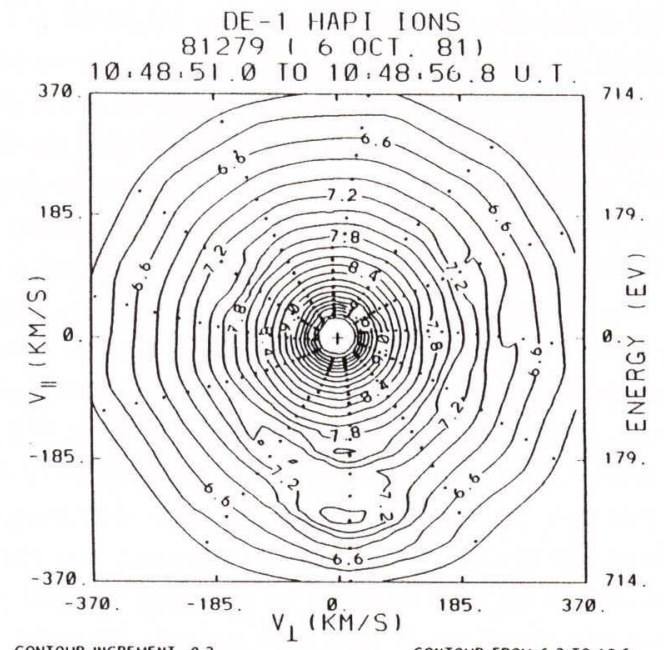

Fig. 10. Contours of the ion distribution function obtained during the same spin of the satellite as in Fig. 8. A field-aligned beam centered in pitch angle near 180 degrees is seen extending to energies of about $500 \mathrm{eV}$.
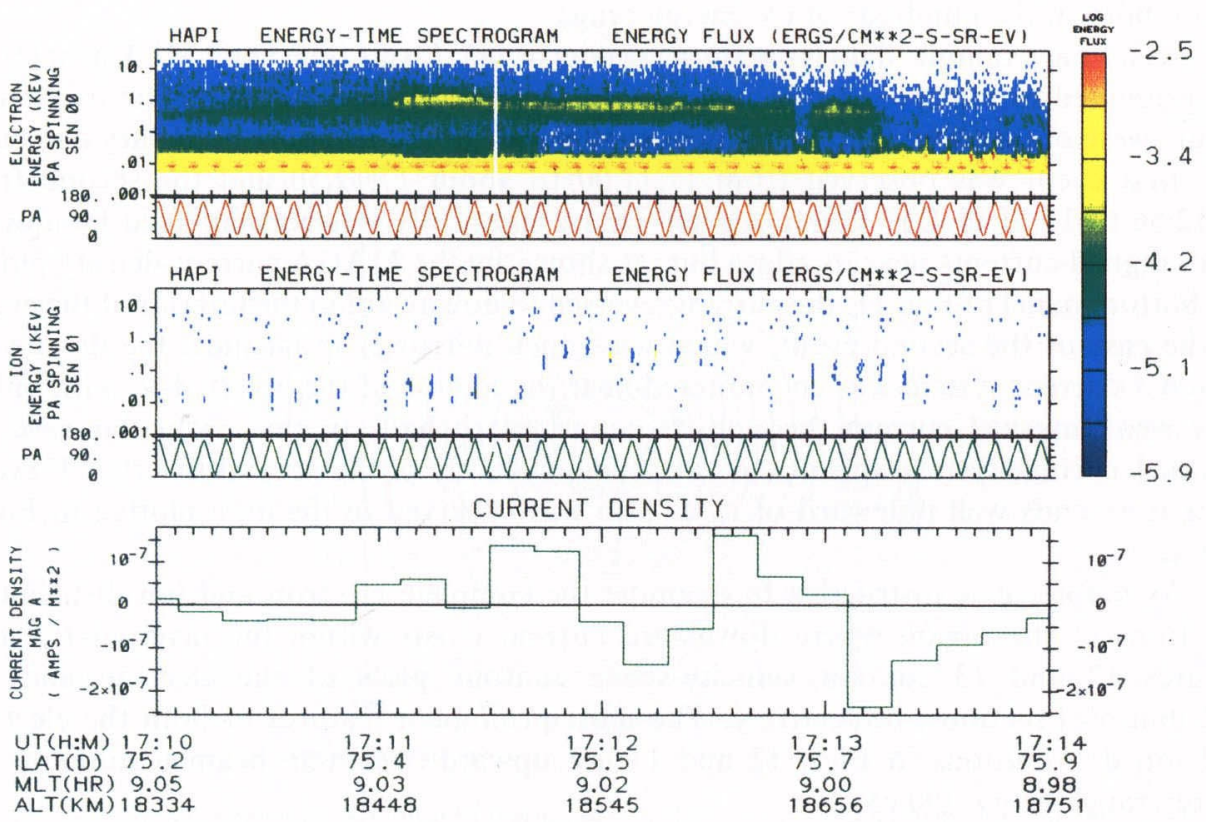

Fig. 11. A three-panel plot of the DE-1 HAPI and MAGA data for a polar pass on day 279 of 1981 in the same format as Fig. 8 but with data at all pitch angles. 
format is shown in Fig. 8 for the period 10:44:30 to 10:54:00 UT. Downward electron energy fluxes are plotted in the upper panel, upward ions in the middle panel, and field-aligned currents (positive upward) derived from the MAGA data in the lower panel. A well-defined inverted- $V$ event appears between 10:46:50 and 10:49:20 UT in Fig. 8, with its poleward boundary coincident with the keV electron trapping boundary. Cusp electron fluxes developed poleward of the trapping boundary, intensifying greatly beginning about 10:51 UT. Upward ion fluxes with energies of a few hundred eV were measured throughout the inverted- $V$, and mirrored cusp ions with energies in the $\mathrm{keV}$ range were seen along with the cusp electrons at higher latitudes. Very weak field-aligned currents were observed until about 10:47:30 UT, when a bifurcated region-1 downward current system was entered and then exited at about 10:49:10 UT. Upward cusp-region field-aligned currents were observed between 10:49:10 and 10:52:45 UT, where another region of downward current was seen. Although such downward currents are often observed in the high-latitude portion of the cusp, they are not the subject of this paper and so will not be discussed in any detail here. Of primary interest to this study is the fact that except for a narrow region of weak upward currents between 10:47:00 and 10:47:24 UT, and again between 10:48:24 and 10:48:36 UT, downward currents were observed throughout the inverted- $V$ event. Figures 9 and 10 contain velocity-space distribution contour plots of electrons and ions, respectively, for one spacecraft spin (about six seconds) within the inverted- $V$ when the field-aligned current was downward. The plots show an upward highly field-aligned electron beam and a somewhat broader upward ion beam, both in the hundreds of $\mathrm{eV}$ energy range.

As a final example, data from the next pass of DE-1, this time at an MLT near 09:00, are presented in Fig. 11. In this case two particularly intense and energetic inverted- $V$ events were observed in the region just equatorward of the trapping boundary and cusp. The first event was observed from 17:11:00 to about 17:12:50 and the second from 17:12:56 to 17:13:15 UT. The first event characteristically was accompanied by upward field-aligned currents near its edges but, as shown by the MAGA current-density plot in the bottom panel of Fig. 11, the current reversed to downward in the interior of the event. In the case of the second event, which was much narrower in latitude, the downward region- 1 current system was encountered near the middle of the inverted- $V$, with only a very weak upward current through its equatorward half. In this particular case the region- 1 current system appears to exist independently of the second inverted- $V$ event, since it extends well poleward of it, as also was observed in the data plotted in Fig. 4 above.

As before, it is instructive to examine the complete electron and ion distribution functions in the region where downward current exists within the inverted- $V$ event. Figures 12 and 13 contain velocity-space contour plots of the electron and ion distribution functions, respectively. The most prominent features of both the electron and ion distributions in Figs. 12 and 13 are upward-streaming beam features in the energy range below $100 \mathrm{eV}$.

\section{Discussion}

The region-1 currents are the primary driving currents for magnetospheric convection. They are generated in the boundary layer (SMIDDY et al., 1980) as the solar wind interacts with the magnetosphere. The quiet-time auroral oval appears as an approxi- 


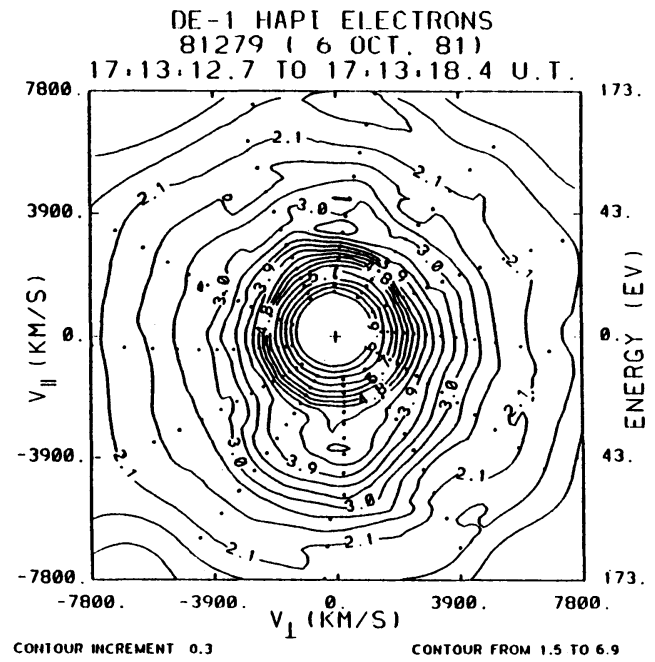

Fig. 12. Contours of the electron distribution function obtained from data taken during one spin of the satellite. Note the beam-like distribution centered near pitch angle 180 and extending in energy to about $50 \mathrm{eV}$.

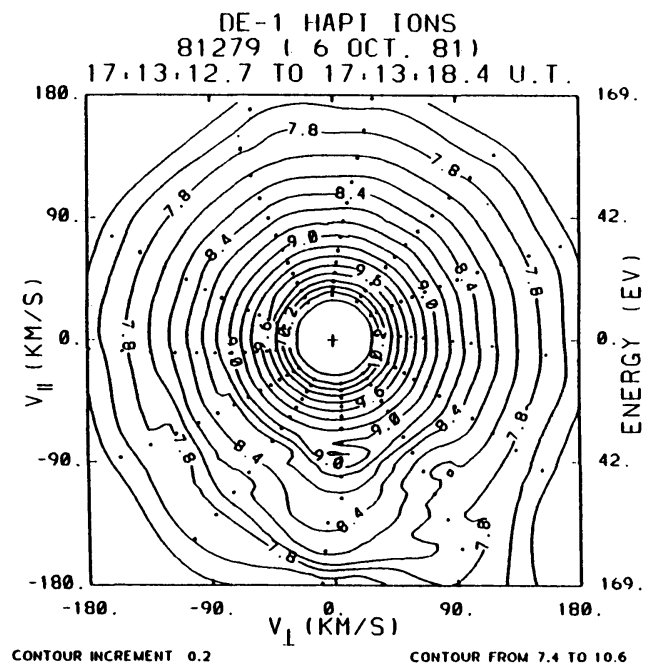

Fig. 13. Contours of the ion distribution function obtained from data taken during the same time as that of Fig. 11. There is a broad (in pitch angle) beam centered near a pitch angle of 180 and extending to energies near $150 \mathrm{eV}$. 
mately continuous circle of discrete auroral arcs. This oval has been variously attributed to the boundary between open and closed magnetic field lines (DESSLER and JUDAY, 1965), the locus of a neutral line encircling the magnetosphere (DUNGEY, 1961), and the locus of upward field-aligned currents (LYONS, 1980). The most obvious inconsistency among these different interpretations concerns the role of the dayside region- 1 current system in the morning sector. If the auroral oval consists of discrete auroral arcs and maps to the boundary layer, then it must be closely related to the region- 1 currents, which flow into the ionosphere in this sector. Moreover, if the region-1 currents are the primary driving currents for magnetospheric convection, then one would expect that they would coincide with the sites of maximum energy dissipation in the ionosphere. The same would not be expected for the region-2 currents or the cusp-region currents. Thus, the assumption that the auroral oval represents the locus of upward field-aligned currents might be called into question by the data presented here, which establish that inverted- $V$ events can exist in regions of downward field-aligned currents. It is now appropriate to identify how this unexpected correlation might occur. We begin by reviewing the important elements of the data that were presented in the previous section and assessing the inferences that may be drawn from each.

The current carriers. The only significant current carriers that have been identified in the DE-1 HAPI data are the downward-moving auroral primary electrons, precipitating cusp electrons, and downward-moving beams of cold electrons (e.g., suprathermal bursts) for the upward currents and upward-accelerated beams of cold electrons for the downward currents. Ion beams are only occasionally and very locally a significant source of field-aligned current. At low altitudes $(<1000 \mathrm{~km}$ or so) the charge carriers of the downward currents are not generally observable with particle detectors, so they are attributed to upward-moving thermal electrons. However, we have shown that at altitudes above about an earth radius significant downward currents result in the acceleration of ionospheric electrons into the tens to hundreds of $\mathrm{eV}$ energy range, rendering them observable to contemporary particle detectors.

As pointed out previously, the upward and downward-moving cold electron beams often appear together as counterstreaming electron distributions (SHARP et al., 1980). However, except for the fluctuating double-layer hypothesis of SHARP et al. (1980), all of the present models for acceleration of ionospheric electrons into the suprathermal-burst type of distribution (JOHNSTONE and WINNINGHAM, 1982) explain only the downwardmoving beams. Although most of the models (e.g., TEMERIN et al., 1986; LOTKO, 1986; MCFADDEN et al., 1987) involve upward parallel electric fields (which would accelerate electrons downward), they all employ wave-particle interactions as the acceleration mechanism for the bursts themselves. This type of acceleration mechanism is also indicated for the upward electron beams that carry the downward region- 1 currents that flow within inverted- $V$ events as shown above.

As was shown in Fig. 5, the electron distribution functions within the dayside inverted- $V$ events contain all of the features of those which have been observed on the nightside and which have been interpreted as evidence for acceleration by quasi-static potential differences above the DE-1 altitudes, which ranged upward to as high as 18,500 $\mathrm{km}$ in the examples shown here. In addition, upward ion beams indicative of further downward electron acceleration below the spacecraft were observed in each case, and these beams were accompanied by upward-moving electron beams wherever the fieldaligned current was downward. There is a possibility that the upward-moving ion 
distributions were actually ion conics, resulting from transverse acceleration at altitudes far below DE-1; however, both the HAPI data and the data from the Energetic Ion Composition Spectrometer (EICS) showed beam, rather than conic, characteristics (W. K. Peterson, private communication). It is thus very unlikely that the upward-moving cold electron beams are also accelerated by quasi-static potential differences. This conclusion agrees with that reached in several previous studies of this type of distribution.

It is not clear, however, how the upward-accelerated cold electron beams can exist above an apparent upward parallel electric field, since such a potential difference would tend to confine the cold ionospheric electrons to low altitudes. In the model proposed by LOTKO (1986) for the production of edge precipitation, cold electrons convect or diffuse into a turbulent region containing a field-aligned potential difference. A similar convection/diffusion process would seem to be necessary for population of the region above the potential drop with cold plasma. Both upward and downward acceleration of the cold electrons by low-frequency waves would then proceed according to the direction of the net field-aligned current in the region. Since there is still another region of inferred potential difference above the altitudes of the DE- 1 observations, a mechanism such as the one proposed by TEMERIN et al. (1986) may be applied to the problem with the additional requirement that upward as well as downward acceleration must be accommodated.

This work was supported by NASA Contract NAS5-33030 and NSF Grant ATM-8805819.

\section{REFERENCES}

BURCH, J. L., Energetic particles and currents: results from dynamics explorer, Rev. Geophys., 26, 215-228, 1988.

BurCh, J. L., Diagnosis of auroral acceleration mechanisms by particle measurements, in Auroral Physics, edited by C.-I. Meng, M. Rycroft, and L. A. Frank, Cambridge Univ. Press, 1990 (in press).

Burch, J. L., P. H. Reiff, R. A. Heelis, J. D. Winningham, W. B. Hanson, C. Gurgiolo, J. D. Menietti, R. A. Hoffman, and J. N. Barfield, Plasma injection and transport in the mid-altitude polar cusp, Geophys. Res. Lett., 9, 921-924, 1982.

Burch, J. L., P. H. ReifF, and M. Sugiura, Upward electron beams measured by DE-1: A primary source of dayside region-1 Birkeland currents, Geophys. Res. Lett., 10, 753-756, 1983.

Chiu, Y. T., A. L. Newman, and J. M. Cornwall, On the structure and mapping of auroral electrostatic potentials, J. Geophys. Res., 86, 10029-10037, 1981.

Chiu, Y. T., J. M. Cornwall, J. F. Fennell, D. J. Gorney, and P. F. Mizera, Auroral plasmas in the evening sector: Satellite observations and theoretical interpretations, Space Sci. Rev., 35, 211-257, 1983.

Dessler, A. J. and R. D. Juday, Configuration of auroral radiation in space, Planet. Space Sci., 13, $63,1965$.

Dungey, J. W., Interplanetary magnetic field and the auroral zones, Phys. Rev. Lett., 6, 47-48, 1961.

Gurgiolo, C. and J. L. Burch, Simulation of electron distributions within auroral acceleration regions, $J$. Geophys. Res., 93, 3989-4003, 1988.

HultQvist, B., On the acceleration of electrons and positive ions in the same direction along magnetic field lines by parallel electric fields, J. Geophys. Res., 93, 9777-9784, 1988.

Hultqvist, B., R. Lundin, K. Stasiewicz, L. Block, P.-A. Lindqvist, G. Gustafsson, H. Koskinen, A. Bahnsen, T. A. Potemera, and L. J. Zanetti, Simultaneous observations of upward moving field-aligned energetic electrons and ions on auroral zone field lines, J. Geophys. Res., 93, 9765-9776, 1988.

Johnstone, A. D. and J. D. Winningham, Satellite observations of suprathermal bursts, J. Geophys. Res., 87 , 2321-2329, 1982.

Klumpar, D. M. and W. J. HeikKILA, Electrons in the ionospheric source cone: Evidence for runaway electrons as carriers of downward Birkeland currents, Geophys. Res. Lett., 9, 873-876, 1982. 
KnIGHT, S., Parallel electric fields, Planet. Space Sci., 21, 741-750, 1973.

Lin, C. S., M. Sugiura, J. L. Burch, J. N. Barfield, and E. Nielsen, DE 1 observations of Type 1 counterstreaming electrons and field-aligned currents, J. Geophys. Res., 89, 8907-8917, 1984.

Lотко, W., Diffusive acceleration of auroral primaries, J. Geophys. Res., 91, 191-203, 1986.

LYONS, L. R., Generation of large-scale regions of auroral currents, electric potentials, and precipitation by divergence of the convection electric fields, J. Geophys. Res., 85, 17-24, 1980.

Marshall, J. A., J. L. Burch, J. R. Kan, and J. A. Slavin, DE 1 observations of return current regions in the nightside auroral oval, J. Geophys. Res., 93, 14542-14548, 1988.

McFadden, J. P., C. W. Carlson, M. H. Boehm, and T. J. Hallinan, Field-aligned electron flux oscillations that produce flickering aurora, J. Geophys. Res., 92, 11,133-11,148, 1987.

MENIETTI, J. D. and J. L. BURCH, DE 1 observations of theta aurora plasma source regions and Birkeland current charge carriers, J. Geophys. Res., 92, 7503-7518, 1987.

OMIDI, N., C. S. Wu, and D. A. GuRnett, Generation of auroral kilometric and Z mode radiation by the cyclotron maser mechanism, J. Geophys. Res., 89, 883-895, 1984.

Sharp, R. D., E. G. Shelley, R. G. Johnson, and A. G. Ghielmetti, Counterstreaming electron beams at altitudes of 1 RE over the auroral zone, J. Geophys. Res., 85, 92-100, 1980.

Smiddy, M., W. J. Burke, M. C. Kelley, N. A. Saflekos, M. S. Gussenhoven, D. A. Hardy, and F. J. RicH, Effects of high-latitude conductivity on observed convection electric fields and Birkeland currents, $J$. Geophys. Res., 85, 6811-6818, 1980.

Temerin, M., J. McFadden, M. Boehm, C. W. Carlson, and W. Lotko, Production of flickering aurora and field-aligned electron flux by electromagnetic ion cyclotron waves, J. Geophys. Res., 91, 5769-5792, 1986. 